\title{
Seeding Strategies for Semantic Disambiguation
}

\author{
Annika Hinze, \\ David Bainbridge \\ University of Waikato \\ Hamilton, New Zealand \\ hinze,davidb@waikato.ac.nz
}

\author{
Extended Abstract \\ Rebekah Wilkins, \\ Craig Taube-Schock \\ University of Waikato \\ Hamilton, New Zealand \\ wilkins,cschock@waikato.ac.nz
}

\author{
J. Stephen Downie \\ University of Illinois \\ Urbana-Champaign, USA \\ jdownie@illinois.edu
}

\begin{abstract}
Semantic disambiguation determines the meaning of words and phrases in a text, for which we use an automatically-generated Concept-in-Context (CiC) network. Words and phrases rarely belong to a single concept; disambiguation in Capisco relies on interplay between words that are in close vicinity in the text. Starting the disambiguation is a seeding process, that identifies the first concepts, which then form the context for further disambiguation steps. This paper introduces the seeding algorithm and explores seeding strategies for identifying these initial concepts in text volumes, such as books, that are stored in a digital library.
\end{abstract}

\section{CCS CONCEPTS}

- Information systems $\rightarrow$ Information systems applications; Digital libraries and archives;

\section{KEYWORDS}

Semantic Disambiguation, Semantic Search, Capisco

\section{ACM Reference Format:}

Annika Hinze, David Bainbridge, Rebekah Wilkins, Craig Taube-Schock, and J. Stephen Downie. 2018. Seeding Strategies for Semantic Disambiguation: Extended Abstract. In $7 C D L$ '18: The 18th ACM/IEEE foint Conference on Digital Libraries, fune 3-7, 2018, Fort Worth, TX, USA. ACM, New York, NY, USA, 2 pages. https://doi.org/10.1145/3197026.3203874

\section{INTRODUCTION}

Semantic Disambiguation determines the meaning (semantic concepts) of words and phrases in a text to allow for subsequent semantic-enhanced search or export of semantic concepts [2]. In Capisco-a semantic disambiguation technique we have devisedthe interplay between words within a certain vicinity of the text is a core feature. Further we use an automatically-generated Conceptin-Context $(\mathrm{CiC})$ network that is created from analyzing Wikipedia link structures. Used together, this means the approach in Capisco can also help, for example, with alleviating issues introduced by OCR-based digitizations, such as used by the HathiTrust Digital Library (HTDL). Starting the disambiguation of words and phrases

Permission to make digital or hard copies of part or all of this work for personal or classroom use is granted without fee provided that copies are not made or distributed for profit or commercial advantage and that copies bear this notice and the full citation on the first page. Copyrights for third-party components of this work must be honored.

For all other uses, contact the owner/author(s).

fCDL '18, fune 3-7, 2018, Fort Worth, TX, USA

(C) 2018 Copyright held by the owner/author(s).

ACM ISBN 978-1-4503-5178-2/18/06

https://doi.org/10.1145/3197026.3203874 in a document is a seeding process that identifies the first concepts, which then form the context for further disambiguation steps throughout the document. This extended abstract introduces the seeding algorithm and explores strategies for identifying these initial concepts. Note that Capisco uses neither machine learning nor natural language processing for disambiguation, but rather relies on co-occurrence of semantically-related words.

\section{SEEDING FOR DISAMBIGUATION}

The disambiguation process has two phases: seeding and full semantic analysis. Both phases use the CiC network, in which Capisco stores triples of literal, concept and context information. The literals

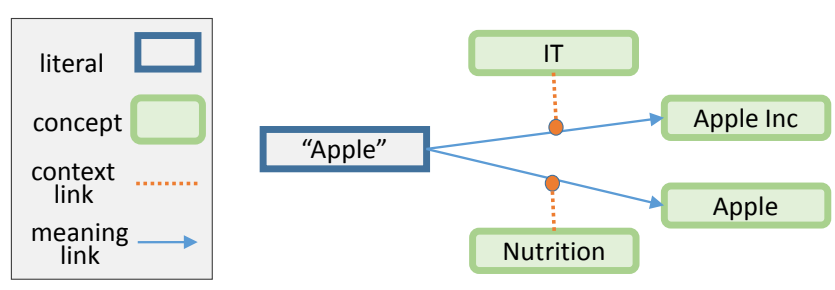

Figure 1: Example CiC triples

refer to words or phrases (e.g., "Apple"), the concepts refers to the meaning of the literal (e.g., [Fruit] or [Computer]), and the contexts refer to the circumstances under which a literal refers to a concept (e.g, [Nutrition] or [IT]), see Figure 1. The above example is described by two triples. The full semantic analysis of terms in a document (introduced in [2]), analyses the presence of other terms in the vicinity. These may, in turn, also be ambiguous but a shared context of these terms may allow for disambiguation. This shared context is determined by the initial seeding.

The seeding for the full disambiguation in Capisco starts by analyzing the stream of tokens from a corpus of text. We focus here on those produced by the OCR analysis of a text. Using the CiC network, Capisco identifies the first 100 terms, i.e., the first 100 phrases or words that can be found as literals in the CiC network. The next step determines the seed concepts by testing if any of these terms have loose semantic connections (vs the stronger connections identified and relied upon in the full semantic analysis). To return to our previous example: let's look at the two terms "Apple" and "Autumn" selected from the 100 terms. We seek to find if they have a loose semantic connection in the $\mathrm{CiC}$ network. This means that the concept [Apple] for the first term "Apple" acts as context for the concept [Autumn] and vice versa. This was identified by 


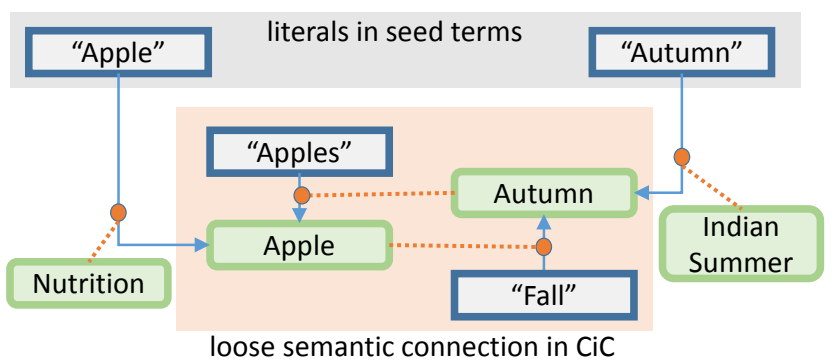

Figure 2: Example of two seed concepts [Apple] \& [Autumn]

searching for all concepts for "Autumn". Figure 2 shows this constellation with the literals occurring in the document's 100 seed terms highlighted in gray and the relevant semantic connections between concepts [Apple] and [Autumn] highlighted in orange. The semantic connection is the stronger the more of those mutual connections exist between two concepts. Note that the the concept/context relationship indicating loose connection (as sufficient for seeding) does not need to be involving those two initial terms. On the other hand, strong semantic connections (suitable for disambiguation) need to use the very terms that appear in the text, but can be spread further apart through the document. The seeding phase concludes once there are either more then 10 connections between seed terms ( $>20$ seed concepts) or at least three pairs of connected seed terms. During the full analysis, these seed concepts and their labels are used to provide (initial) contexts for identifying the meaning of terms encountered.

Scanned books such as those provided by the HTDL typically contain a number of OCR artifacts throughout (i.e., misreading of characters, manual annotations) but often at the beginning of a volume (due to cover patterns and soiled pages). Restriction to seed terms of at least 3 char length removes errors (such as " $\mathrm{ft}$ " and " $\mathrm{mm}$ " from cover patterns being identified as [Feet] and [Metre]). Among the first clearly discernible terms in many OCR-ed documents are references to digitization (e.g., "digitized", "Internet archive"). These types of terms are well supported in Wikipedia (and thus in the CiC) and potentially create an overwhelming number of seed concepts, and therefore need to be excluded.

\section{SEEDING STRATEGIES}

Capisco's seeding process as described above has successfully been used for page-based disambiguation (seeding per page). As this creates a large overhead and does not carry forward any previously found seeds, it is most appropriate for documents in which each page contains distinct information (e.g., poetry, encyclopedias). For volume-based analysis discussed here, the seeding process starts at the title page.

Varied seed base: Titles often contain terms that are strongly represented in Wikipedia, which leads to a narrow set of seed concepts as they all circle around very similar topics (e.g., 9 different grammars and 12 languages among 30 seeds) for [3]. Instead of allowing each seed term to participate in more than one semantic connection, we progressed through the seed terms once a match had been found, which led to a greater variation of seed concepts.

Extracted Features: We used the 100 most significant nouns of each volume as seed terms, based on Part-of-Speech tagging on unigrams (enforces copyright protection) [4]. Term significance was identified using AntConc [1]. Unfortunately, the restriction to unigrams prevents recognition of significant terms such as "Isle of Man” [3] or person names.

Metadata: We used curated volume metadata [4] as seed terms, combining title, author and publisher information. We added any names that were provided in normalized first-name surname form. As this data does not contain any OCR errors and with names matching Wikipedia's naming convention, this strategy provided best results.

\section{CONCLUSION}

This paper explored seeding strategies for volume-based semantic disambiguation in digital libraries based on the Wikipedia linking structure. Our test results for a set of 100 out-of-copyright volumes from the HTDL is available on github (https://git.io/vN9lE).

\section{REFERENCES}

[1] Laurence Anthony. 2018. (2018). corpus analysis toolkit, at www.laurenceanthony. net/software/antconc/.

[2] Annika Hinze, Craig Taube-Schock, David Bainbridge, Rangi Matamua, and J. Stephen Downie. 2015. Improving Access to Large-scale Digital Libraries Through Semantic-enhanced Search and Disambiguation. In ACM/IEEE FCDL'15. ACM, 147-156.

[3] J. Kelly. 1870. A Practical Grammar of the Antient Gaelic, Or Language of the Isle of Man, Usually Called Manks. Quaritch, London.

[4] HathiTrust Digital Library. 2018. (2018). extracted feature set, at https://wiki. htrc.illinois.edu/display/COM/Extracted+Features+Dataset. 